\title{
Protective effect of proanthocyanidins on anoxia-reoxygenation injury of myocardial cells mediated by the PI3K/Akt/GSK-3 $\beta$ pathway and mitochondrial ATP-sensitive potassium channel
}

\author{
YANYAN HU ${ }^{1}$, LI LI ${ }^{2}$, WENBIN YIN ${ }^{1}$, LIN SHEN ${ }^{1}$, BEIAN YOU $^{1}$ and HAIQING GAO ${ }^{1}$ \\ ${ }^{1}$ Department of Geriatrics, Qilu Hospital of Shandong University; ${ }^{2}$ Department of Medicine, \\ Shandong University, Jinan, Shandong 250012, P.R. China
}

Received August 30, 2013; Accepted April 9, 2014

DOI: $10.3892 / \mathrm{mmr} .2014 .2459$

\begin{abstract}
The aim of the present study was to examine the protective effect of proanthocyanidins anoxia-reoxygenation injury of myocardial cells and its association with phosphatidylinositol-3-kinase/Akt and glycogen synthase kinase (PI3K/Akt/GSK)-3 $\beta$ and ATP-sensitive potassium channels. Neonatal rat myocardial cells were cultured and an anoxia-reoxygenation model was established following pretreatment with various drugs. The experiment was divided into five groups according to an experimental scheme. An MTT assay was used to examine the cell survival, and reactive oxygen species (ROS) levels and apoptosis were detected by flow cytometry. Myocardial apoptosis was also examined by terminal deoxynucleotidyl transferase dUTP nick end labeling staining and western blot analysis was employed to detect the expression levels of caspase-3, p-Akt and p-glycogen synthase kinase (GSK)-3 3 . The results revealed that myocardial cells in the anoxia-reoxygenation group (A/R) exhibited reduced survival rates, increased ROS levels and enhanced caspase-3 expression, as compared with the control group $(\mathrm{CN} ; \mathrm{P}<0.05)$. However, the increase in p-Akt and p-GSK-3 $\beta$ expression was not significantly different. In the proanthocyanidin pretreatment group (PC) the myocardial cell survival rate was increased, ROS levels were reduced, caspase-3 expression was decreased and p-Akt and p-GSK- $3 \beta$ expression levels were significantly increased as compared with the $\mathrm{A} / \mathrm{R}$ group $(\mathrm{P}<0.05)$. Blockade of the PIK3/Akt channel by LY294002 eliminated the protective effects of proanthocyanidins and induced a significant decrease in $\mathrm{p}-\mathrm{Akt}$ protein and $\mathrm{p}-\mathrm{GSK}-3 \beta$ expression levels as compared with the PC group. The inhibitor of mitochondrial ATP-sensitive potassium (mitoKATP) channels, 5-HD, also
\end{abstract}

Correspondence to: Professor Haiqing Gao, Department of Geriatrics, Qilu Hospital of Shandong University, 107 Culture West Road, Jinan, Shandong 250012, P.R. China

E-mail: gaohaiqingdoctor@yeah.net

Key words: anoxia-reoxygenation, apoptosis, PI3K/Akt/GSK-3 $\beta$ signaling pathway, mitochondrial ATP-sensitive potassium channel significantly suppressed the protective effects of proanthocyanidins, but had no evident impact on p-Akt and p-GSK-3 $\beta$ expression as compared with the PC group. In conclusion, pretreatment with proanthocyanidins had a protective effect on rat myocardial cell anoxia/reoxygenation injury. This effect is associated with the activation of the PI3K/Akt/GSK-3 $\beta$ signaling pathway and the opening of mitoKATP channels, which may have important roles downstream of PI3K.

\section{Introduction}

Coronary atherosclerosis heart disease (coronary heart disease) is a leading cause of mortality in the 21 st century, among which acute myocardial infarction (AMI) is one of the most serious clinical manifestations associated with high occurrence rate, high mortality and poor long-term prognosis $(1,2)$. The early, continuous and full opening of infarct-associated vessels to recover blood perfusion in the ischemic myocardium is the most important principle of treatment for AMI. In recent years, with the wide application of intravenous thrombolysis, percutaneous coronary interventions (PCI), coronary artery bypass grafting and other strategies, there has been an evident improvement in the symptoms and prognosis for patients with coronary heart disease and AMI (3). However, despite the numerous strategies available, it is common that clinical symptoms do not improve as expected. On the contrary, numerous patients emerge with reperfusion arrhythmia, increased myocardial infarction area and cardiac insufficiency. As a result, the concept of myocardial ischemia reperfusion injury has emerged. Attempting to relieve the occurrence of reperfusion injury has become a prominent obstacle in the prevention and treatment of ischemic heart disease.

Myocardial ischemia reperfusion injury is a highly complex process, and currently there are several key pathogenic mechanisms that are considered to be involved, including oxidative stress injury, intracellular calcium overload, cell apoptosis, cellular energy loss and activation of neutrophil inflammatory reaction (4). Ischemia reperfusion injury also involves multiple regulatory mechanisms, including the activation and inactivation of signaling pathways (5). In processes including ischemic pre-adaptation, ischemic post-adaptation and pharmacological pretreatment, the confluence of numerous pathways is 
triggered by the heart to release endogenous active substances, activating various intracellular signal transduction systems to adjust cardiac function, which has a protective effect on the myocardium (5).

Proanthocyanidins are highly efficient free radical scavengers, which are widely used in the clinic to delay senility, regulate blood fat and to reduce the development of atherosclerosis and tumor growth (6). Several studies have identified that proanthocyanidins have a protective effect on myocardial ischemia reperfusion, but the endogenous mechanism underlying this effect is has yet to be elucidated. In the present study, myocardial cells cultured in vitro were exposed to acute anoxia-reoxygenation in order to simulate myocardial ischemia-reperfusion injury. Pretreatment with proanthocyanidins was performed to study their ability to relieve anoxia-reoxygenation injury and to investigate the specific manifestation mode of this injury. Furthermore, it was investigated whether the protective effects of proanthocyanidins on anoxia-reoxygenation injury in myocardial cells proceeded via the phosphatidylinositol-3-kinase/Akt and glycogen synthase kinase (P13K/Akt/GSK)-3 $\beta$ signaling pathway and the mitochondrial ATP potassium (mitoKATP) channel.

\section{Materials and methods}

Experimental animals and reagents. Sprague Dawley rats, aged 1-3 days, were provided by the Animal Center of Shandong University (Jinan, Shandong, China). This study was approved by the Ethics Committee of Shandong University (Jinan, China). Several regents were used, including Dulbecco's modified Eagle's medium (DMEM; Invitrogen Life Technologies, Carlsbad, CA, USA), trypsin (Tiangen Biotech (Beijing) Co., Ltd., Beijing, China), dichlorofluorescein probe (Sigma, St. Louis, MO, USA), MTT proliferation detection kit (Sigma, San Jose, CA, USA), a terminal deoxynucleotidyl transferase dUTP nick end labeling (TUNEL) kit (Tiangen Biotech (Beijing) Co., Ltd.), Annexin V-fluorescein isothiocyanate (FITC) cell apoptosis detection kit (Invitrogen Life Technologies), LY294002 and 5-hydroxy decanoic acid (5-HD; Sigma , CA, USA), mouse anti-rat Akt antibody (Santa Cruz Biotechnology, Inc., Santa Cruz, CA, USA). Furthermore, mouse anti-rat glycogen synthase kinase (GSK)-3 $\beta$ and caspase- 3 antibodies were purchased from Cell Signaling Technology, Inc. (Danvers, MA, USA).

Isolation of neonatal cardiomyocytes. Isolation of neonatal cardiomyocytes is a technically more simple procedure than cell isolation from adult hearts, as it does not require aorta cannulation and perfusion. A two-step procedure was employed, consisting of enzyme digestion and mechanical agitation of the ventricular tissue followed by purification of the cardiomyocyte population. The enzymatic digestion and purification of cardiomyocytes was performed as previously described (7).

Cell culture. For the culture of neonatal myocardial cells, two methods were employed, including a re-differentiation and a rapid attachment method as previously described $(8,9)$. The redifferentiation method was performed to ensure that the neonatal cells regained a typical morphology and shape, concurrent with slow cell attachment to the substrate. The rapid attachment method resulted in an improved retention of in vivo myocyte morphology and functionality, as well as ease of use in experiments.

Establishment of the anoxia-reoxygenation model. The experiment was started when the myocardial cells grew close to confluence, exhibiting synchronous growth cycles. Ischemia simulation solution components included $\mathrm{NaH}_{2} \mathrm{PO}_{4} 0.9 \mathrm{mmol} / 1, \mathrm{NaHCO}_{3} 6.0 \mathrm{mmol} / 1, \mathrm{CaCl}_{2}$ $1.8 \mathrm{mmol} / 1, \mathrm{MgSO}_{4} 1.2 \mathrm{mmol} / 1$, sodium lactate $40 \mathrm{mmol} / 1$, 4-(2-hydroxyethyl)-1-piperazineethanesulfonic acid (HEPES) $20 \mathrm{mmol} / \mathrm{l}, \mathrm{NaCl} 98.5 \mathrm{mmol} / \mathrm{l}, \mathrm{KCl} 10.0 \mathrm{mmol} / \mathrm{l}$ at $\mathrm{pH}$ 6.8. Furthermore, cells were presaturated in an atmosphere of $95 \% \mathrm{~N}_{2}$ and $5 \% \mathrm{CO}_{2}$ for $1 \mathrm{~h}$ to create an oxygen pressure of $\mathrm{P}\left(\mathrm{O}_{2}\right) \leq 4.0 \mathrm{Kpa}$, to allow the cells to contain high concentrations of $\mathrm{K}^{+}$, lactic acid and $\mathrm{H}^{+}$, a low oxygen concentration, and no glucose or other energy substrates. Cultured cells were kept in this anoxia solution under anoxic conditions for $3 \mathrm{~h}$. The solution was then replaced with reoxygenation solution containing $\mathrm{NaH}_{2} \mathrm{PO}_{4} 0.9 \mathrm{mmol} / \mathrm{l}, \mathrm{NaHCO}_{3} 20 \mathrm{mmol} / \mathrm{l}$, $\mathrm{CaCl}_{2} 1.0 \mathrm{mmol} / \mathrm{l}, \mathrm{MgSO}_{4} 1.2 \mathrm{mmol} / \mathrm{l}$, glucose $5.5 \mathrm{mmol} / \mathrm{l}$, HEPES $20 \mathrm{mmol} / \mathrm{l}, \mathrm{NaCl} 129.5 \mathrm{mmol} / \mathrm{l}, \mathrm{KCl} 5.0 \mathrm{mmol} / \mathrm{l}$, and the $\mathrm{pH}$ was adjusted to the reoxygenation condition of $95 \%$ oxygen saturation for $3 \mathrm{~h}$.

Trial grouping. The present study was divided into five groups according to an experimental scheme. The control group $(\mathrm{CN})$ consisted of myocardial cells cultured under normal conditions. The anoxia-reoxygenation group (AR) was composed of the anoxia/reoxygenation injury model in myocardial cells subjected to anoxia for $3 \mathrm{~h}$ and reoxygenation for $3 \mathrm{~h}$. In the proanthocyanidin (Shenfu Inc., Shanxi, China) pretreatment groups (PC), the culture medium was added with a final concentration of $100 \mathrm{mg} / \mathrm{l}$ of the proanthocyanidins and the cells were incubated for $2 \mathrm{~h}$ prior to exposure to anoxic and reoxygenation conditions. In the LY294002 (blocker of the PIK3/Akt channel) group, culture medium was added containing LY294002 at concentration of $15 \mu \mathrm{mol} / 1$, proanthocyanidins were added $30 \mathrm{~min}$ later, then anoxia and reoxygenation were conducted. In the 5-HD [an inhibitor of mitochondrial ATP-sensitive potassium (mitoKATP) channels] group, the culture medium was added to 5-HD at a concentration of $100 \mu \mathrm{mol} / 1$, proanthocyanidins were added $30 \mathrm{~min}$ later and then anoxia and reoxygenation were conducted.

Measurement of reactive oxygen species (ROS). ROS levels were measured by flow cytometry. Following treatment, cells were trypsinized and centrifuged ( $2 \mathrm{~min}$ at 20,000 x g), and the cell pellet was treated with 2',7'-dichlorodihydrofluorescein diacetate stain $(1: 200)$, resuspended and incubated at $37^{\circ} \mathrm{C}$ for $20 \mathrm{~min}$ in the dark. Cells not treated with $2^{\prime}, 7^{\prime}$-dichlorodihydrofluorescein diacetate were used as negative controls and stained cells treated with $100 \mu l$ hydrogen peroxide $(30 \% \mathrm{w} / \mathrm{v}$ hydrogen peroxide) incubated for $10 \mathrm{~min}$ served as positive controls. ROS levels were measured using a flow cytometer (Becton-Dickinson, Franklin Lakes, NJ, USA) and quantified by determining the mean fluorescence for each treatment. 


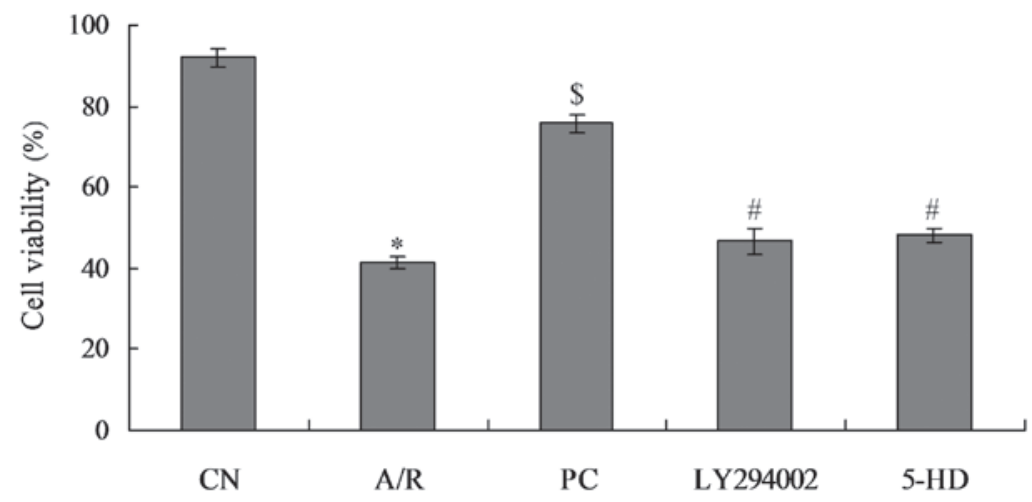

Figure 1. Cell viability in groups ( $\mathrm{n}=10$, mean \pm standard deviation). ${ }^{*} \mathrm{P}<0.05$ for $\mathrm{A} / \mathrm{R}$ compared with the $\mathrm{CN}$ group; ${ }^{\mathrm{P}} \mathrm{P}<0.05$ for $\mathrm{PC}$ compared with the $\mathrm{A} / \mathrm{R}$ group and ${ }^{\#} \mathrm{P}<0.05$ for LY294002 (blocker of the PIK3/Akt channel) and 5-HD (inhibitor of mitochondrial ATP-sensitive potassium channels) compared with the PC group. CN, control group; A/R, anoxia-reoxygenation group; PC, the proanthocyanidins pretreatment group; 5-HD, 5-hydroxy decanoic acid; PIK3, phosphatidylinositol-3-kinase.

TUNEL assay. Potential DNA fragmentation was examined by the TUNEL apoptosis detection kit (Chemicon, Temecula, CA, USA). The specific procedure of the TUNEL assay was performed as previously described (10).

Flow cytometric analysis. Binding of annexin V-FITC and uptake of propidium iodide (PI) into the cells were assessed using a FACScan flow cytometer (Becton-Dickinson). Briefly, cells were harvested, resuspended and incubated with Annexin V-FITC and PI $(5 \mu \mathrm{g} / \mathrm{ml})$ in the dark at room temperature for $15 \mathrm{~min}$. Fluorescence was measured through a 530/30 band filter (FL-1) to monitor Annexin V-FITC binding and through a 585/42 band filter (FL-2) to monitor PI uptake.

Western blot analysis. Western blotting was employed to analyze the expression levels of caspase-3, p-Akt, GSK-3 $\beta$ and $\mathrm{p}-\mathrm{GSK}-3 \beta$. The assay was performed as previously described (11).

Statistical analysis. Data were expressed as the mean \pm standard deviation (SD), variance analysis was used to compare multiple groups and the q test was used to analyze inter-group differences. Analysis was performed using SASS 6.12 statistical software (SPSS, Inc., Chicago, IL, USA) and P<0.01 was considered to indicate a statistically significant difference between values.

\section{Results}

Myocardial cell survival rate in each group. Myocardial cells were evidently impaired in the A/R group $(41.33 \pm 1.45 \%)$, and the cell survival rate was significantly reduced as compared with that in the control group $(91.95 \pm 2.27 \%$; $\mathrm{P}<0.05)$. Cell survival rate in the $\mathrm{PC}$ group $(75.64 \pm 2.01 \%)$, was significantly higher than that in the $\mathrm{A} / \mathrm{R}$ group $(\mathrm{P}<0.05)$. The cell survival rate in the LY294002 group $(46.56 \pm 3.3 \%)$ was significantly lower than that in the PC group $(\mathrm{P}<0.05)$ as compared with that in $\mathrm{A} / \mathrm{R}$ group, where there were no significant differences. The cell survival rate in the 5-HD group $(48.17 \pm 1.7 \%)$ was significantly lower than that in the PC group $(\mathrm{P}<0.05)$, while there was no significant difference from that in the $A / R$ group. There was no significant difference between the cell survival rate in the LY294002 group and that in the 5-HD group (Fig. 1).

ROS levels in myocardial cells in each treatment group. In the A/R group (fluorescence intensity, 211.69), ROS levels were significantly increased as compared with those in the $\mathrm{CN}$ group (27.06; $\mathrm{P}<0.01)$. ROS levels were decreased in the $\mathrm{PC}$ group (80.11) as compared with those in the A/R group, which were significantly different $(\mathrm{P}<0.01)$. ROS levels were significantly increased in the LY294002 group (180.45) as compared with those in the $\mathrm{PC}$ group $(\mathrm{P}<0.01)$, while there was no significant difference from those in the $A / R$ group. ROS levels increased significantly in the 5-HD group (168.35) as compared with those in the $\mathrm{PC}$ group $(\mathrm{P}<0.01)$; however, there was no significant difference from those in the $A / R$ group. There were no significant differences between the ROS level of cells in the LY294002 and 5-HD groups (Fig. 2).

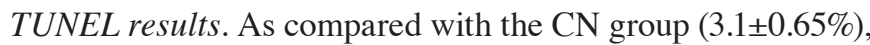
the percentage of TUNEL-positive cells in the A/R group $(25.3 \pm 2.64 \%)$ was significantly increased $(\mathrm{P}<0.05)$. As compared with the A/R group, TUNEL-positive cells in the PC group $(10.2 \pm 1.59 \%)$ were significantly decreased $(\mathrm{P}<0.01)$. TUNEL-positive cells in the LY294002 group (18.6+1.79\%) were significantly increased as compared with those in the PC group $(\mathrm{P}<0.01)$. TUNEL-positive cells in the 5-HD group $(19.5 \pm 2.03 \%)$ were also significantly increased when compared with the PC group $(\mathrm{P}<0.01)$. There were no significant differences between the A/R and the 5-HD groups, between the LY294002 and 5-HD groups and between the LY294002 and A/R groups (Fig. 3).

Apoptotic rates of myocardial cells. Flow cytometric analysis was used to detect the Annexin V/FITC and PI staining and determine apoptotic rates. In the Fig. 4, cells in the bottom right of the dot plot represent cells in late apoptosis, while those in the top right of the graph represent cells in early apoptosis. Apoptotic cells in the A/R group (30.70\%) were significantly increased as compared with those in the $\mathrm{CN}$ group $(8.36 \%$; $\mathrm{P}<0.01)$. The percentage of apoptotic cells was significantly decreased in the PC group (13.11\%) as compared with that in the $\mathrm{A} / \mathrm{R}$ group $(\mathrm{P}<0.01)$. Furthermore, apoptotic 

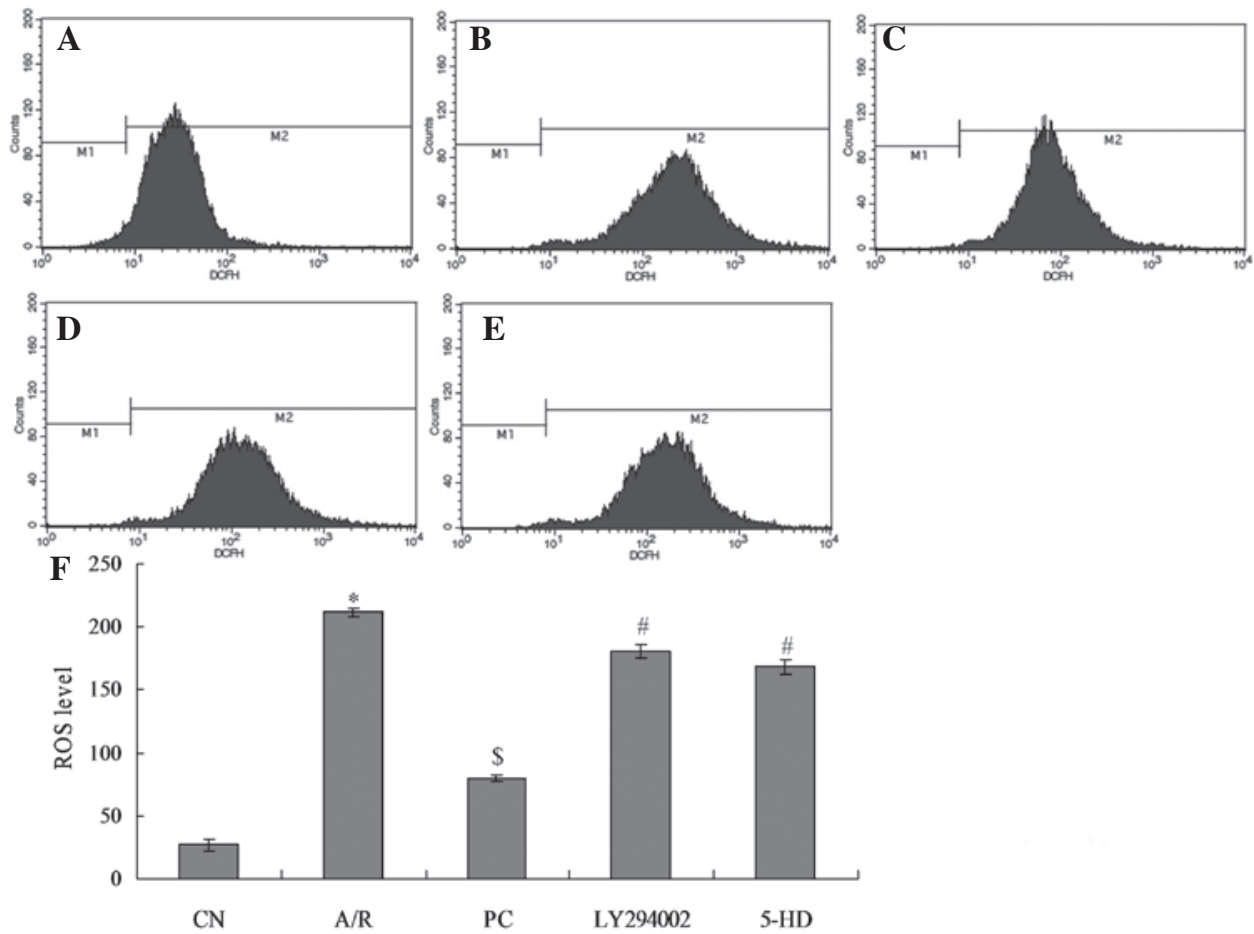

Figure 2. ROS levels in each group ( $\mathrm{n}=10, \pm \mathrm{s}$ ). (A) CN group; (B) A/R group; (C) PC group; (D) LY294002 (blocker of the PIK3/Akt channel) group; (E) 5-HD (inhibitor of mitoKATP channels) group. (F) Statistical analysis of the ROS levels in each group. ${ }^{*} \mathrm{P}<0.05$ for $\mathrm{A} / \mathrm{R}$ compared with the $\mathrm{CN}$ group; ${ }^{\text {\$ }} \mathrm{P}<0.05$ for $\mathrm{PC}$ compared with the A/R group and ${ }^{~} \mathrm{P}<0.05$ for LY294002 and 5-HD compared with the PC group. CN, control group; A/R, anoxia-reoxygenation group; $\mathrm{PC}$, the proanthocyanidin pretreatment group; 5-HD, 5-hydroxy decanoic acid; PIK3, phosphatidylinositol-3-kinase.
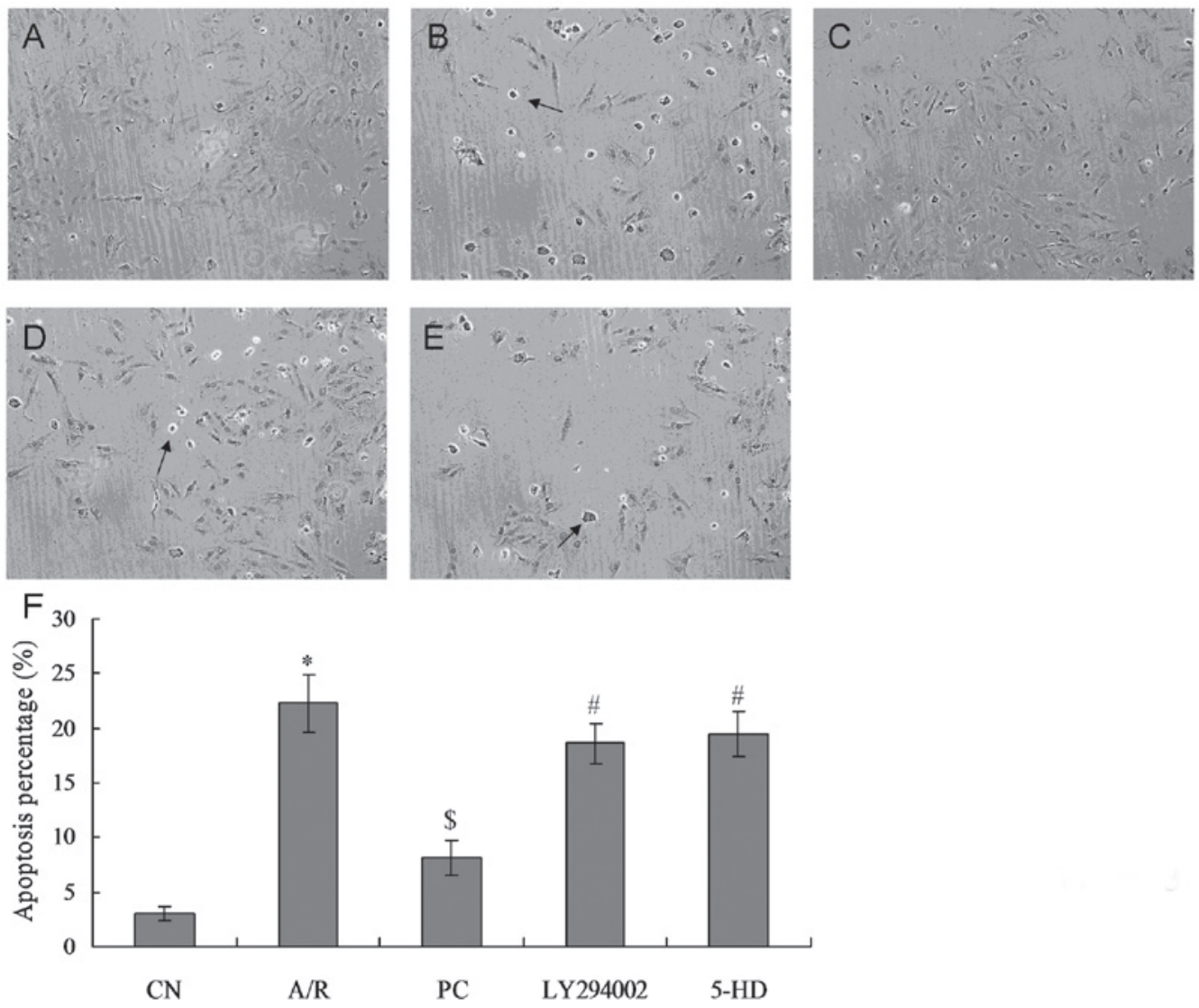

Figure 3. Analysis of TUNEL-positive cells in each group. (A) CN group; (B) A/R group; (C) PC group; (D) LY294002 (blocker of the PIK3/Akt channel) group; (E) 5-HD (inhibitor of mitoKATP channels) group. Magnification, x200. (F) Statistical analysis of the TUNEL-positive cells. "P<0.05 for A/R compared with the $\mathrm{CN}$ group; ${ }^{\$} \mathrm{P}<0.05$ for PC compared with the A/R group and ${ }^{*} \mathrm{P}<0.05$ for $\mathrm{LY} 294002$ and 5 -HD compared with the PC group. CN, control group; $\mathrm{A} / \mathrm{R}$, anoxia-reoxygenation group; PC, the proanthocyanidins pretreatment group; 5-HD, 5-hydroxy decanoic acid; PIK3, phosphatidylinositol-3-kinase; TUNEL, terminal deoxynucleotidyl transferase dUTP nick end labeling. 

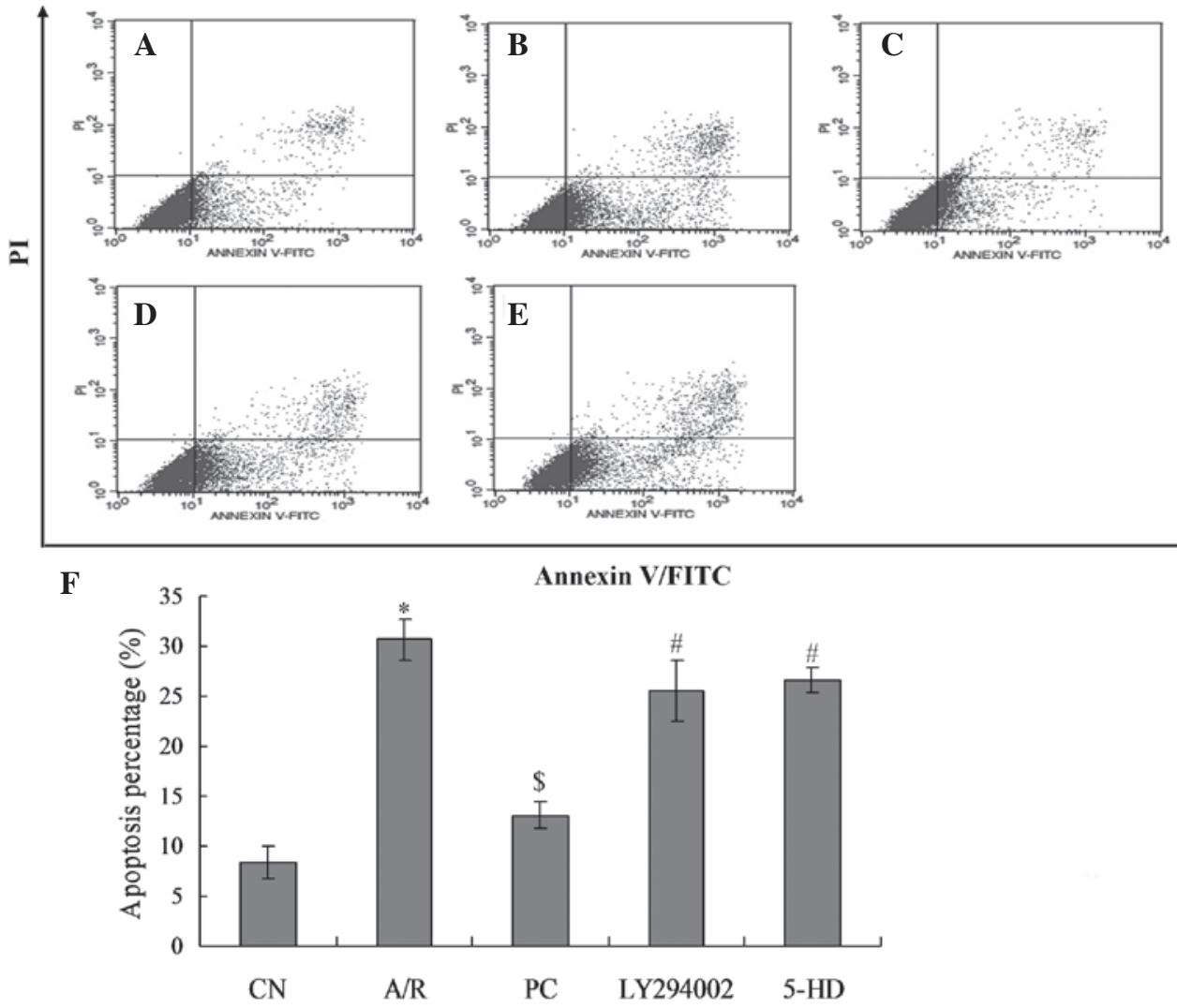

Figure 4. Flow cytometric analysis of Annexin V/FITC and PI double staining. (A) CN group; (B). A/R group; (C) PC group; (D) LY294002 (blocker of the PIK3/Akt channel) group; (E) 5-HD (inhibitor of mitoKATP channels) group. (F) Statistical graph of annexin V/FITC and PI staining. "P $<0.05$ for A/R compared with the $\mathrm{CN}$ group; ${ }^{\$} \mathrm{P}<0.05$ for PC compared with the A/R group and ${ }^{\#} \mathrm{P}<0.05$ for LY294002 and 5-HD compared the with PC group. PI, propidium iodide; CN, control group; A/R, anoxia-reoxygenation group; PC, the proanthocyanidins pretreatment group; 5-HD, 5-hydroxy decanoic acid; PIK3, phosphatidylinositol-3-kinase; FITC, fluorescein isothiocyanate.

A

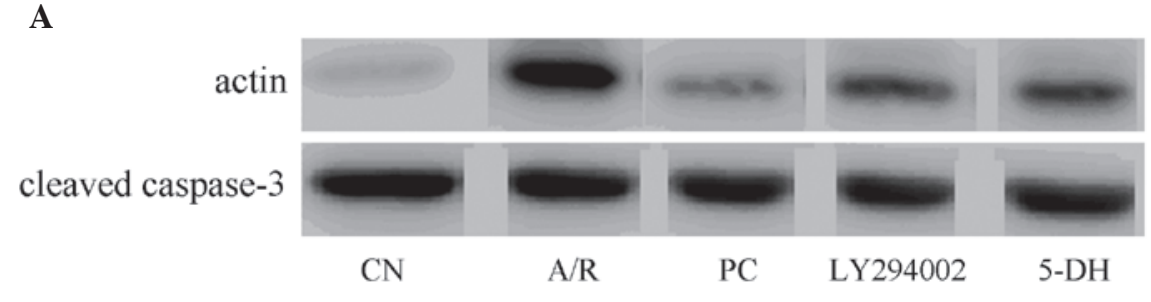

B

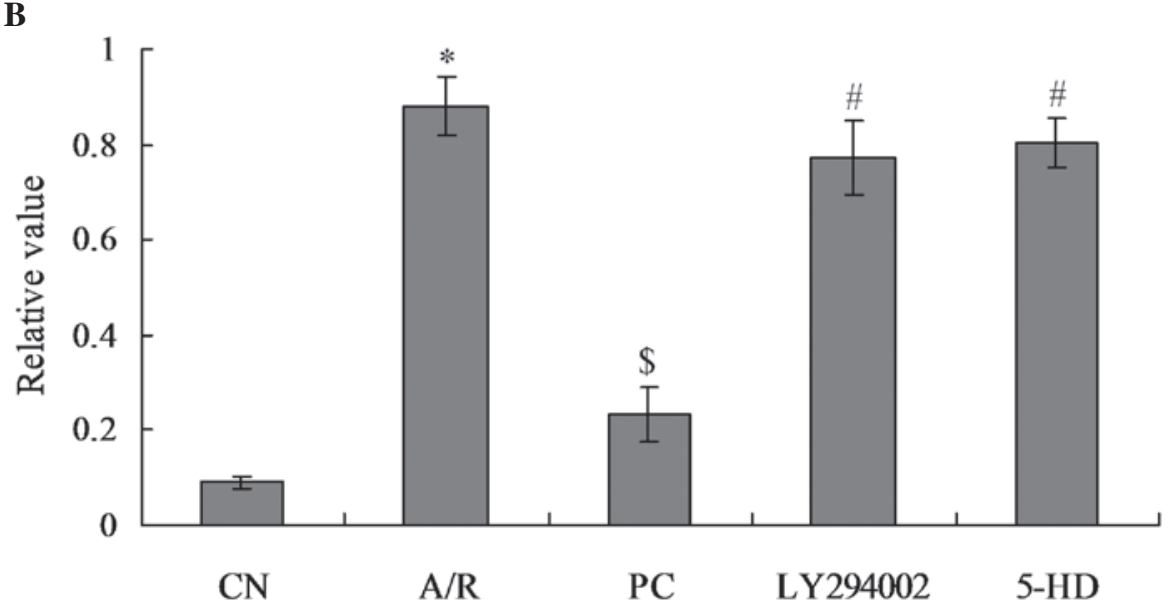

Figure 5. Western blot analysis results of cleaved caspase-3 in each group. (A) Western blot analysis. (B) Statistical graph of western blotting bands. The relative value of each preparation is calculated by each gray numerical value of specific product vs that of $\beta$-actin. " $\mathrm{P}<0.05$ for $\mathrm{A} / \mathrm{R}$ compared with the $\mathrm{CN}$ group; ${ }^{\$} \mathrm{P}<0.05$ for PC compared with the A/R group and ${ }^{\#} \mathrm{P}<0.05$ for LY294002 (blocker of the PIK3/Akt channel) and 5-HD (inhibitor of mitoKATP channels) compared with the PC group. CN, control group; A/R, anoxia-reoxygenation group; PC, the proanthocyanidins pretreatment group; 5-HD, 5-hydroxy decanoic acid; PIK3, phosphatidylinositol-3-kinase. 
A
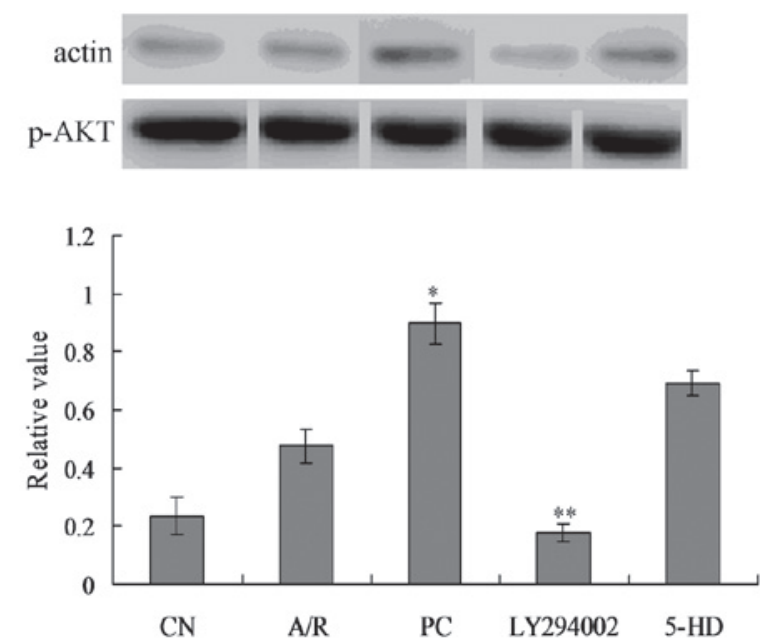

B
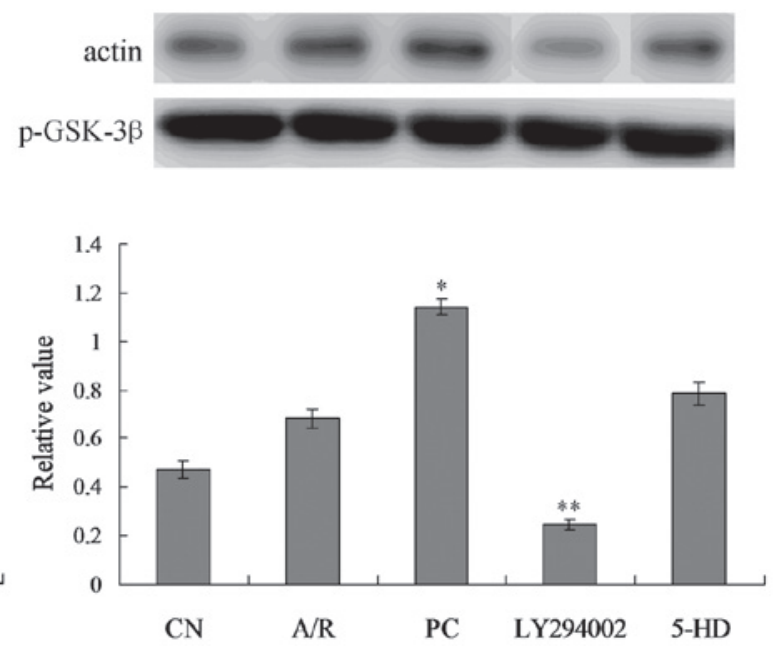

Figure 6. p-Akt and p-GSK-3 $\beta$ expression in each group. (A) p-Akt expression and statistical analysis. (B) p-GSK-3 $\beta$ expression and statistical analysis. The relative value of each preparation was calculated by each gray numerical value of specific product vs that of $\beta$-actin. " $\mathrm{P}<0.05$ for $\mathrm{A} / \mathrm{R}$ compared with the $\mathrm{CN}$ group and ${ }^{* *} \mathrm{P}<0.01$ for LY294002 (blocker of the PIK3/Akt channel) compared with the PC group. GSK, glycogen synthase kinase; CN, control group; A/R, anoxia-reoxygenation group; PC, the proanthocyanidins pretreatment group; 5-HD, 5-hydroxy decanoic acid; PIK3, phosphatidylinositol-3-kinase.

cells in the LY294002 (25.48\%) and 5-DH groups were significantly increased when compared with those in the PC group $(\mathrm{P}<0.05)$. There was no significant difference between the LY294002 and 5-DH groups. Furthermore, the percentage of apoptotic cells in the LY294002 and 5-DH groups demonstrated no significant difference when compared with that in the $\mathrm{A} / \mathrm{R}$ group $(\mathrm{P}>0.05)$.

Activated caspase-3 levels in each group. The levels of activated caspase-3 protein (cleaved caspase-3) reflected the degree of apoptosis in myocardial cells, which showed a positive correlation. From Fig. 5, it was identified that caspase-3 in the $\mathrm{A} / \mathrm{R}$ group were significantly activated as compared with those in the $\mathrm{CN}$ group $(\mathrm{P}<0.05)$. When treated with proanthocyanidins, caspase-3 activation was significantly inhibited in the PC group, and activated caspase-3 level was significantly decreased, compared with the $\mathrm{A} / \mathrm{R}$ group $(\mathrm{P}<0.05)$. Furthermore, activated caspase-3 level in the LY294002 and 5 -DH groups was significantly increased, when compared with the PC group $(\mathrm{P}<0.05)$. However, there was no significant difference between the LY294002 and 5-DH groups.

$p$-Akt and $p$-GSK-3 $\beta$ expression analysis. p-Akt protein expression was moderately increased in the A/R group, but there was no significant difference when compared with the $\mathrm{CN}$ group (Fig. 6A). p-Akt protein expression increased significantly in PC group when compared with the A/R group $(\mathrm{P}<0.05)$. p-Akt protein expression decreased significantly in the LY294002 group when compared with the PC group $(\mathrm{P}<0.05)$. There was no significant difference between the PC and 5-DH group $(\mathrm{P}>0.05)$. Furthermore, there was no significant difference of t-Akt expression between every group (data not shown).

p-GSK-3 $\beta$ protein expression was slightly increased in the $\mathrm{A} / \mathrm{R}$ group, but there was no significant difference when compared with the $\mathrm{CN}$ group (Fig. 6B). p-Akt protein expression increased significantly in the PC group when compared with the A/R group $(\mathrm{P}<0.05)$. $\mathrm{p}-\mathrm{GSK}-3 \beta$ protein expression decreased significantly in LY294002 group when compared with the PC group $(\mathrm{P}<0.05)$. There was no significant difference in $\mathrm{p}-\mathrm{GSK}-3 \beta$ expression between $\mathrm{PC}$ group and $5-\mathrm{DH}$ group $(\mathrm{P}>0.05)$. Furthermore, there was no significant difference of t-GSK-3 $\beta$ among every group (data not shown).

\section{Discussion}

Oxidative stress is important in myocardial anoxia-reoxygenation injury, where excessive ROS production is derived from the mitochondrial electron transport chain, activated neutrophils and the enzyme xanthine oxidase $(12,13)$. ROS have a highly potent chemical activity, and their active electrons easily militate against the components of cells, enhancing cell membrane lipid peroxidation, restraining the function of cellular proteins and damaging nucleic acids and chromosomes, resulting in overall damage to cellular structure and functioning with harmful consequences $(14,15)$. In the present study, it has been identified that the myocardial cell survival rate decreased following anoxia-reoxygenation injury, ROS production was enhanced and the apoptotic rate of myocardial cells increased. Cell apoptosis, also known as programmed cell death (PCD), is induced by the activation of signal transduction pathways, which trigger karyocyte-dependent activation of endogenous DNA incision enzymes. Under certain physiological or pathological conditions, this process is able to independently regulate gene expression, inducing characteristic morphological and biochemical changes to normal cellular functioning. Caspase-3 has a key function in the implementation of apoptosis. Apoptotic stimuli induce a reduction in the mitochondrial transmembrane potential, triggering cytochrome $\mathrm{C}$ release into the cytoplasm and the subsequent activation of a caspase-mediated apoptosis reaction, which drives the rapid execution of cell death (16). Apoptosis is critical in the pathological process underlying myocardial ischemia-reperfusion injury. Zhao et al (17) identi- 
fied that inhibiting myocardial apoptosis significantly reduced the area of myocardial infarction, by not only relieving the necrotic lesion of ischemia-reperfusion, but also by improving the mechanical contractile function of the heart.

When the mitochondrial outer membrane ruptures and the mitochondrial content, including apoptosis inducing factor (AIF) and cytochrome $\mathrm{C}$, is released, this disrupts the electron transfer chain and directly induces cell death $(18,19)$. Through the study of the association between ROS generation and the opening of the mitochondrial permeability transition pore (mPTP) during the process of myocardial anoxia-reoxygenation injury with apoptosis, it has been identified that the generation of ROS occurs earlier than the opening of the mPTP. This indicates that ROS may cause the opening of the mPTP, as single anoxia does not induce the opening of the mPTP. However, large amounts of ROS are generated following reoxygenation and the MPTP opens. A rapid and wide opening of the MPTP may cause excessive expansion of mitochondria and uncoupling of oxidative phosphorylation, which causes hydrolysis of large amounts of ATP, leading to cell death (20). Accordingly, stabilizing the permeability transition pore in the mitochondrial membrane and reducing the production of ROS are important in preventing cellular apoptosis. Bergmann et al (21) identified that pravastatin pretreatment reduces the generation of ROS in anoxia-reoxygenation-injured myocardial cells, as well as the rate of cellular apoptosis. The results of the present study revealed that proanthocyanidins effectively inhibited the production of ROS induced by acute anoxia-reoxygenation, which subsequently inhibited apoptosis. These data are consistent with earlier studies, confirming that the antiapoptotic effect of proanthocyanidins proceeds via inhibition of the mitochondrial pathway, which reduces caspase-3 activity.

The PI3K/Akt/GSK-3 $\beta$ signalling pathway is the most important signal transduction pathway in myocardial ischemic pretreatment. The protective effect of ischemic pretreatment on the myocardium is predominantly due to the activation of the PI3K/Akt signal transduction pathway (22). Efthymiou et al (23) identified that administering atorvastatin reduced the myocardial infarction area (MI) in the ischemia reperfusion model of the normal myocardium of rats in vitro. Furthermore, these effects were eliminated by the PI3K/Akt inhibitor, indicating that the protective effect of atorvastatin on the myocardium was mediated by the PI3K/Akt pathway, inhibiting apoptosis and regulating the transport of glucose in correlation with glycogen synthesis. In addition, studies have demonstrated that GSK-3 $\beta$ is associated with the opening of the MPTP.

In the myocardial cells of the anoxia-reoxygenation model, the addition of diazoxide prior to cell anoxia reduced intracellular calcium overload, mitochondrial membrane potential and oxidative stress induced-apoptosis. By contrast, the addition of the mitoKATP channel blocker 5-HD eliminated this protective effect (24). One study revealed that oxytocin is protective against myocardial ischemia-reperfusion injury by reducing the area of myocardial infarction and myocardial enzyme release, and this effect was inhibited by 5-HD and atractyloside (a mPTP-opening agent). ROS produced in the process of anoxia-reoxygenation regulate the permeability of the MPTP and thus reduce apoptosis, while mitoKATP regulates the production of ROS (25). The mitoKATP channel has a protective role in anoxia-reoxygenation injury; however, the precise location of interference with the process remains to be identified. A number of studies have hypothesized that the mitoKATP channel is the initiating factor in the protection mechanism of ischemia-reperfusion injury, while others consider it to be the final effector (26-28).

Previous studies have identified that proanthocyanidins have a protective effect on myocardial ischemia reperfusion. Sato et al (29) demonstrated that grape seed extract promotes the recovery of cardiac contractile function following ischemia and reperfusion, as a significant reduction in the myocardial infarct area was observed and it appeared to directly scavenge peroxyl radicals, thus leading to the inhibition of cell apoptosis. In one study, proanthocyanidins attenuated the isoproterenol-induced activation of various enzymes in the mitochondria, including lysosomal enzyme, isocitrate dehydrogenase, cytochrome $\mathrm{C}$ oxidase, NADH dehydrogenase, and reduced the area of myocardial infarction (30). In the present study, myocardial cells were exposed to anoxia-reoxygenation conditions in vitro in order to simulate myocardial ischemia-reperfusion injury. This model did not consider the effect of neural, humoral and other hybrid cell factors present in in vivo and in vitro perfusion models. Following proanthocyanidin treatment, the myocardial cells exhibited an increase in overall survival, a reduction in ROS levels and a decrease in the apoptotic rate, all of which showed significant differences. These data demonstrated that pretreatment with proanthocyanidins had a protective effect on myocardial cells with anoxia-reoxygenation injury. The addition of the PI3K/Akt-specific inhibitor LY294002 and the mitoKATP channel-specific blocker 5-HD prior to pretreatment with proanthocyanidins eliminated the protective effects of proanthocyanidin, including the reduction in myocardial intracellular ROS and apoptotic rate. These data suggested that the PI3K/Akt/GSK-3 $\beta$ signaling pathway and mitoKATP channels were activated by the proanthocyanidins, which are likely to be the mechanisms underlying the protective effect of proanthocyanidins on myocardial anoxia-reoxygenation injury. Following the addition of the P13K blocker, the protein levels of $\mathrm{p}$-Akt and $\mathrm{p}-\mathrm{GSK}-3 \beta$ were significantly reduced, which were significantly different when compared with those in the PC group. Following administration of the mitoKATP channel-specific blocker, PI3K and phosphorylated GSK-3 $\beta$ showed no significant difference, which indicated that the mitoKATP channel may have an important role downstream of the P13K/Akt/GSK-3 $\beta$ pathway.

In conclusion, pretreatment with proanthocyanidins had a protective effect on myocardial cell anoxia-reoxygenation injury in rat cells. This effect was associated with the activation of the PI3K/Akt/GSK-3 $\beta$ signaling pathway and the opening of mitoKATP channels, which may have an important role downstream of the PI3K pathway.

\section{Acknowledgements}

This study was supported by a grant from the Independent Innovation Foundation of Shandong University (grant no. 2012TS153). 


\section{References}

1. Zhang LN, Liu PP, Zhou J, Huang RS, Yuan F, Fei LJ, Huang Y, $\mathrm{Xu}$ L, Hao LM, Qiu XJ, et al: Positive correlation between variants of lipid metabolism-related genes and coronary heart disease. Mol Med Rep 8: 260-266, 2013.

2. Zhang S, Liu X, Goldstein S, Li Y, Ge J, He B, Fei X, Wang Z and Ruiz G: Role of JAK/STAS signaling pathway in the pathogenesis of acute myocardial infarction in rats and its effect on NF- $\kappa B$ expression. Mol Med Rep 7: 93-98, 2013.

3. Rao SV, Hess CN, Dai D, Green CL, Peterson ED and Douglas PS: Temporal trends in percutaneous coronary intervention outcomes among older patients in the United States. Am Heart J 166: 273-281, 2013.

4. Wang N, Min , Li D, He P and Zhao L: Geranylgeranylacetone protects against myocardial ischemia and reperfusion injury by inhibiting high-mobility group box 1 protein in rats. Mol Med Rep 5: 521-524, 2012.

5. Turer AT and Hill JA: Pathogenesis of myocardial ischemia-reperfusion injury and rationale for therapy. Am J Cardiol 106: 360-368. 2010

6. Xu Z, Du P, Meiser P and Jacob C: proanthocyanidins: oligomeric structures with unique biochemical properties and great therapeutic promise. Nat Prod Commun 7: 381-388, 2012.

7. Louch WE, Sheehan KA and Wolska BM: Methods in cardiomyocyte isolation, culture, and gene transfer. J Mol Cell Cardiol 51: 288-298, 2011.

8. Cantin M, Ballak M, Beuzeron-Mangina J, Anand-Srivastava MB and Tautu C: DNA synthesis in cultured adult cardiocytes. Science 214: 569-570, 1981.

9. Piper HM, Probst I, Schwartz P, Hütter FJ and Spieckermann PG: Culturing of calcium stable adult cardiac myocytes. J Mol Cell Cardiol 14: 397-412, 1982.

10. Wang X, Dong CF, Shi Q, Shi S, Wang GR, Lei YJ, Xu K, An R, Chen JM, Jiang HY, et al: Cytosolic prion protein induces apoptosis in human neuronal cell SH-SY5Y via mitochondrial disruption pathway. BMP Rep 42: 444-449, 2009.

11. Wang W, Shi Q, Xu K, Gao C, Chen C and Li XL, et al: Familial CJD associated PrP mutants within the transmembrane region induced Ctm-PrP retention in ER and trigger apoptosis by ER stress in SH-SY5Y cells. PLoS One 6: e14602, 2011.

12. Wang HJ, Kang PF, Wu WJ, Tang Y, Pan QQ, Ye HW, Tang B, $\mathrm{Li} \mathrm{ZH}$ and Gao Q: Changes in cardiac mitochondrial aldehyde dehydrogenase 2 activity in relation to oxidative stress and inflammatory injury in diabetic rats. Mol Med Rep 8: 686-690, 2013.

13. Decoursey TE and Ligeti E: Regulation and termination of NADPH oxidase activity. Cell Mol Life Sci 62: 2173-2193, 2005.

14. Marczin N, E1-Habashi N, Hoare GS, Bundy RE and Yacoub M: Antioxidants in myocardial ischemia-reperfusion injury: therapeutic potential and basic mechanisms. Arch Biochem Biophys 420: 222-236, 2003.

15. Yang H, Fan S, Song D, Wang Z, Ma S, Li S, Li X, Xu M, Xu M and Wang X: Long-term streptozotocin-induced diabetes in rats leads to severe damage of brain blood vessels and neurons via enhanced oxidative stress. Mol Med Rep 7: 431-440, 2013.
16. Scarabelli TM, Stephanou A, Pasini E, et al: Minocycline inhibits caspase activation and reactivation, increases the ratio of $\mathrm{XIAP}$ to smac/DIABLO, and reduces the mitochondrial leakage of cytochrome C and smac/DIABLO. J Am Coll Cardiol 43: 865-874, 2004.

17. Zhao ZQ, Morris CD, Budde JM, Wang NP, Muraki S and Sun HY: Inhibition of myocardial apoptosis reduces infarct size and improves regional contractile dysfunction during reperfusion. Cardiovasc Res 59: 132-142, 2003.

18. Arguad L, Gateau-Roesh O, Raisky O, Loufouat J, Robert D and Ovize M: Postconditioning inhibits mitochondrial permeability transition. Circulation 111: 194-197, 2005.

19. Yang $X$ and Huang N: Berberine induces selective apoptosis through the AMPK-mediated mitochondrial/caspase pathway in hepatocellular carcinoma. Mol Mep Rep 8: 505-510, 2013.

20. Assaly R, de Tassigny AD, Paradis S, Jacquin S, Berdeaux A and Morin D: Oxidative stress, mitochondrial permeability transition pore opening and cell death during hypoxia-reoxygenation in adult cardiomyocytes. Eur J Pharmacol 675: 6-14, 2012.

21. Bergmann MW, Rechner C, Freund C, Baurand A, El Jamali A and Dietz R: Statins inhibit reoxygenation-induced cardiomyocyte apoptosis: role for glycogen synthase kinase 3 beta and transcription factor beta-catenin. J Mol Cell Cardiol 37: 681-690, 2004.

22. Liu ZL, Mao JH, Peng AF, Yin QS, Zhou Y, Long XH and Huang SH: Inhibition of fatty acid synthase suppresses osteosarcoma cell invasion and migration via downregulation of the PI3K/Akt signaling pathway in vitro. Mol Med Rep 7: 608-612, 2013.

23. Efthymiou CA, Mocanu MM and Yellon DM: Atorvastatin and myocardial reperfusion injury: new pleiotropic effect implicating multiple prosurvival sinaling. J Cardiovasc Pharmac 45: 247-252, 2005.

24. Abdallah Y, Wolf C, Meuter K, Piper HM, Reusch HP and Ladilov Y: Preconditioning with diazoxide prevents reoxygenation-induced rigor-type hypercontracture. J Mol Cell Cardiol 48: 270-276, 2010.

25. Alizadeh AM, Faghihi M, Khori V, Sohanaki H, Pourkhalili K, Mohammadghasemi F and Mohsenikia M: Oxytocin protects cardiomyocytes from apoptosis induced by ischemia-reperfusion in rat heart: role of mitochondrial ATP-dependent potassium channel and permeability transition pore. Peptides 36: 71-77, 2012.

26. Grossini E, Pollesello P, Bellofatto K, et al: Protective effects elicited by levosimendan against liver ischemia/reperfusion injury in anesthetized rats. Liver Transpl 20: 361-375, 2014.

27. Amani M, Jeddi S, Ahmadiasl N, Usefzade $\mathrm{N}$ and Zaman J: Effect of HEMADO on level of CK-MB and LDH enzymes after ischemia/reperfusion injury in isolated rat heart. Bioimpacts 3: 101-104, 2013.

28. Zeng Z, Huang HF, He F, et al: Diazoxide attenuates ischemia/reperfusion injury via upregulation of heme oxygenase-1 after liver transplantation in rats. World J Gastroenterol 18: 1765-1772, 2012.

29. Sato M, Maulik G, Ray PS, Bagchi D and Das DK: Cardioprotective effects of grape seed proanthocyanidin against ischemic reperfusion injury. J Mol Cell Cardiol 31: 1289-1297, 1999.

30. Liu X, Qiu J, Zhao S, You B, Ji X, Wang Y, Cui X, Wang Q and Gao H: Grape seed proanthocyanidin extract alleviates ouabain-induced vascular remodeling through regulation of endothelial function. Mol Med Rep 6: 949-954, 2012. 